\title{
Cardiac macrophages regulate isoproterenol-induced Takotsubo-like cardiomyopathy
}

\author{
Xudong Liao, ${ }^{1}$ Eugene Chang, ${ }^{1}$ Xinmiao Tang, ${ }^{1,2}$ Ippei Watanabe, ${ }^{1}$ Rongli Zhang, ${ }^{1}$ Hyun-Woo Jeong, ${ }^{3}$ \\ Ralf H. Adams, ${ }^{3}$ and Mukesh K. Jain ${ }^{1}$ \\ ${ }^{1}$ Case Cardiovascular Research Institute, Case Western Reserve University School of Medicine, Harrington Heart and \\ Vascular Institute, University Hospitals Cleveland Medical Center, Cleveland, Ohio, USA. ${ }^{2}$ Yueyang Hospital, Shanghai \\ University of Traditional Chinese Medicine, Shanghai, China. ${ }^{3}$ Max Planck Institute for Molecular Biomedicine, Department \\ of Tissue Morphogenesis, Münster, Germany.
}

Takotsubo syndrome (TTS) is an acute, stress-induced cardiomyopathy that occurs predominantly in women after extreme physical and/or emotional stress. To date, our understanding of the molecular basis for TTS remains unknown and, consequently, specific therapies are lacking. Myocardial infiltration of monocytes and macrophages in TTS has been documented in clinical studies. However, the functional importance of these findings remains poorly understood. Here, we show that a single high dose of isoproterenol (ISO) in mice induced a TTS-like cardiomyopathy phenotype characterized by female predominance, severe cardiac dysfunction, and robust myocardial infiltration of macrophages. Single-cell RNA-Seq studies of myocardial immune cells revealed that TTS-like cardiomyopathy is associated with complex activation of innate and adaptive immune cells in the heart, and macrophages were identified as the dominant immune cells. Clobal macrophage depletion (via clodronate liposome administration) or blockade of macrophage infiltration (via a CCR2 antagonist or in CCR2-KO mice) resulted in recovery of cardiac dysfunction in ISO-challenged mice. In addition, damping myeloid cell activation by HIF1 $\alpha$ deficiency or exposure to the immunomodulatory agent bortezomib ameliorated ISO-induced cardiac dysfunction. Collectively, our findings identify macrophages as a critical regulator of TTS pathogenesis that can be targeted for therapeutic gain.

Authorship note: XL and EC contributed equally to this work.

Conflict of interest: The authors have declared that no conflict of interest exists.

Copyright: (c) 2022, Liao et al. This is an open access article published under the terms of the Creative Commons Attribution 4.0 International License.

Submitted: October 28, 2021

Accepted: December 15, 2021 Published: February 8, 2022

Reference information: /CI Insight. 2022;7(3):e156236.

https://doi.org/10.1172/jci.

insight.156236.

\section{Introduction}

Takotsubo syndrome (TTS), also known as broken heart syndrome, is an acute, stress-induced cardiomyopathy that occurs after extreme physical and/or emotional stress (1). The majority ( $90 \%)$ of patients with TTS are older women (aged $>60$ years) (2). TTS confers an in-hospital mortality rate similar to that of acute coronary syndromes $(3,4)$. In survivors, the recurrence rate is $2 \%$ to $4 \%$ annually up to $20 \%$ at 10 years, with a $50 \%$ rate of major adverse cardiac and cerebrovascular events despite cardiac recovery (5). TTS symptoms can be similar to those of a myocardial infarction (MI) (5), including chest pain, dyspnea, abnormal ECG (ST elevation), elevated plasma cardiac troponin level, and left ventricular wall motion abnormalities (LVWMAs). However, distinct from acute coronary syndrome, patients with TTS do not demonstrate coronary blockade and the LVWMAs do not follow a regional pattern. Therapy for TTS is largely supportive and agents such as beta blockers, which confer survival benefit in the setting of MI, are largely ineffective in patients with TTS $(6,7)$. To date, our understanding of the molecular basis for TTS remains unknown and, consequently, specific therapies are lacking (8).

Intense emotional and/or physical stress can trigger TTS; therefore, hyperactivation of the sympathetic nervous system (SNS) is a leading hypothesis about the mechanism of TTS $(2,8)$. Indeed, it has been shown that plasma levels of both epinephrine and norepinephrine were markedly increased during the acute phase of TTS to levels many times higher than that occurring with MI $(5,9)$, suggesting a pathogenic role of adrenergic signaling in TTS. Consistent with this idea, clinical conditions associated with high catecholamine levels, including pheochromocytoma, thyrotoxicosis, and subarachnoid hemorrhage with sympathetic storm, have been reported to cause TTS (10-12). Perhaps the most robust evidence is from cohorts 
of patients whose TTS was triggered by administration of $\beta$-agonists, either for medical reasons or by accident (13). Similarly, in several animal models, administration of high-dose epinephrine or isoproterenol (ISO) has been reported to induce TTS-like acute reversible left ventricular (LV) dysfunction (14).

Recent clinical studies (via biopsy specimens and MRI) have documented that TTS is characterized by a myocardial infiltration of monocytes and macrophages $(15,16)$. But whether these macrophages play a pathogenic role in TTS is unknown. Macrophages are found in virtually every tissue and are critical in the regulation of homeostasis and stress-induced responses. Macrophages broadly consist of 2 classes: tissue-resident macrophages and blood-borne infiltrating macrophages. At steady state, the adult heart contains 2 major subsets of $\mathrm{Ly}^{6} \mathrm{C}^{-} / \mathrm{CCR} 2^{-}$resident macrophages that originate from yolk-sac erythromyeloid progenitors (17). In addition, there are 2 minor subsets of $\mathrm{Ly} 6 \mathrm{C}^{+}$macrophages that are derived from hematopoietic stem cells and maintained through monocytes infiltration (18). Upon myocardial injury, cardiac macrophage numbers increase via both resident macrophage proliferation and monocyte infiltration, which is important for the progression and resolution of tissue injury (19-21). Specific markers of cardiac resident macrophages, such as Tim4 (T cell membrane protein 4, also known as TIMD4 [T cell immunoglobulin and mucin domain containing 4]) and Lyve1 (lymphatic vessel endothelial hyaluronan receptor 1), have been identified in recent studies, enabling in vivo mapping of cardiac macrophage subsets (22).

Here, we show that a single high dose of ISO induced a severe TTS-like cardiomyopathy phenotype characterized by female predominance, reversible severe cardiac dysfunction, and robust myocardial infiltration of macrophages. Using pharmacologic and genetic mouse models, we demonstrate that reducing macrophage infiltration or macrophage activation can ameliorate ISO-induced cardiac dysfunction. One of the pharmacologic agents used is an FDA-approved immunomodulatory agent; thus, these findings have immediate translational implications.

\section{Results}

ISO induced TTS-like cardiomyopathy in female mice. The pathophysiology of TTS is thought to be driven by high levels of catecholamines that are released by SNS in response to extreme emotional or physical stress $(5,9)$. We first established a mouse model mimicking TTS by administering a single dose of ISO by i.p. injection. To assess TTS-like responses, cardiac function was assessed by determining left ventricular ejection fraction (LVEF; by transthoracic echocardiography at baseline [before injection], 1 day after injection [acute TTS], and 3, 7, and 10 days after injection [recovery]). Dose-titration studies were undertaken in female and male mice, with the primary readout being significant cardiac dysfunction. In female WT mice, a $200 \mathrm{mg} / \mathrm{kg}$ dose triggered LV dysfunction without death by day 7, whereas 400 $\mathrm{mg} / \mathrm{kg}$ ISO resulted in 100\% mortality after 3 days (Supplemental Figure 1A; supplemental material available online with this article; https://doi.org/10.1172/jci.insight.156236DS1). In contrast, male mice showed more variability in response to ISO (Supplemental Figure 1B). The potential reason for different TTS-like outcomes in female and male mice might be due to sex differences in sensitivity to adrenergic stimulation (23). Therefore, we used female mice administered $200 \mathrm{mg} / \mathrm{kg}$ ISO i.p. for subsequent studies. Since most patients with TTS are women $(\sim 90 \%)$, our model with female mice is consistent with the clinical setting.

After a single dose of ISO, female mice consistently exhibited acute LV dysfunction (LVEF <35\%) at 1 day and nearly complete recovery (LVEF $>60 \%$ ) by 7 days (Figure 1, A and B). Of note, we confirmed ISO-induced LV apical ballooning (Figure 1A), recapitulating the classic clinical TTS phenotype. Other LV akinetic regions were also observed, with lower frequency. Consistent with clinical observations, ISO induced elevation of plasma cardiac troponin (cTnT) levels, a biomarker of myocardial injury (Figure 1C). Notably, despite LVEF recovery at 7 days after ISO administration, cTnT levels remained high, suggesting ongoing myocardial injury from a transient surge of catecholamine. Histological studies revealed sporadic regions of severe myocardial injury by day 7 after ISO injection, coupled with fibrosis and cell death in the same regions (Figure 1D). However, such injuries were not observed at earlier time points, indicating ISO-induced acute cardiac dysfunction preceded cardiac remodeling. Collectively, these findings suggest a single high dose of ISO successfully induced TTSlike cardiomyopathy in female mice.

ISO administration induces significant myocardial infiltration of macrophages. TTS myocardium is characterized with accumulation of monocytes and macrophages (15), indicating myocardial inflammation plays a role in TTS. Therefore, we subjected ISO-challenged mouse hearts to FACS to determine if ISO 
A

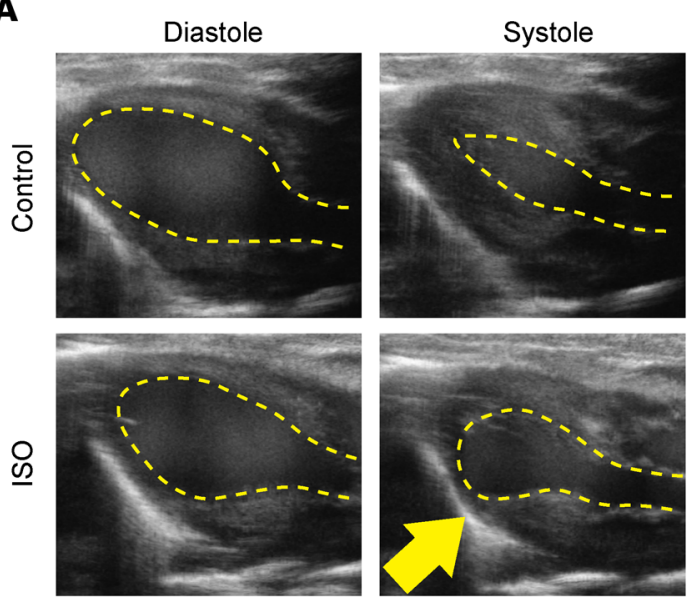

B

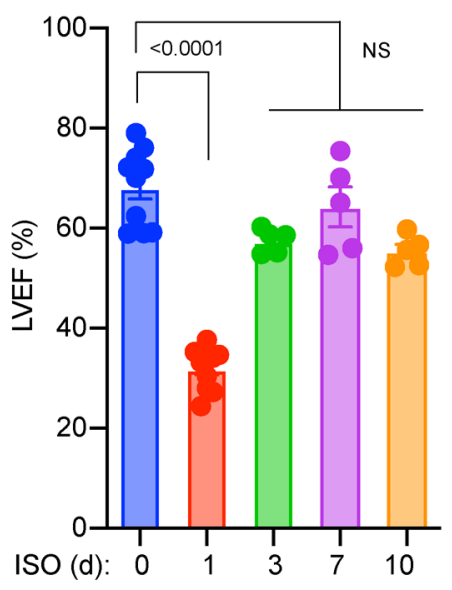

C

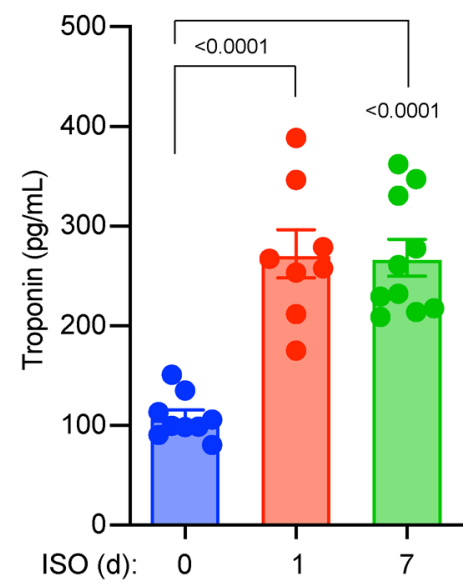

ISO 7d
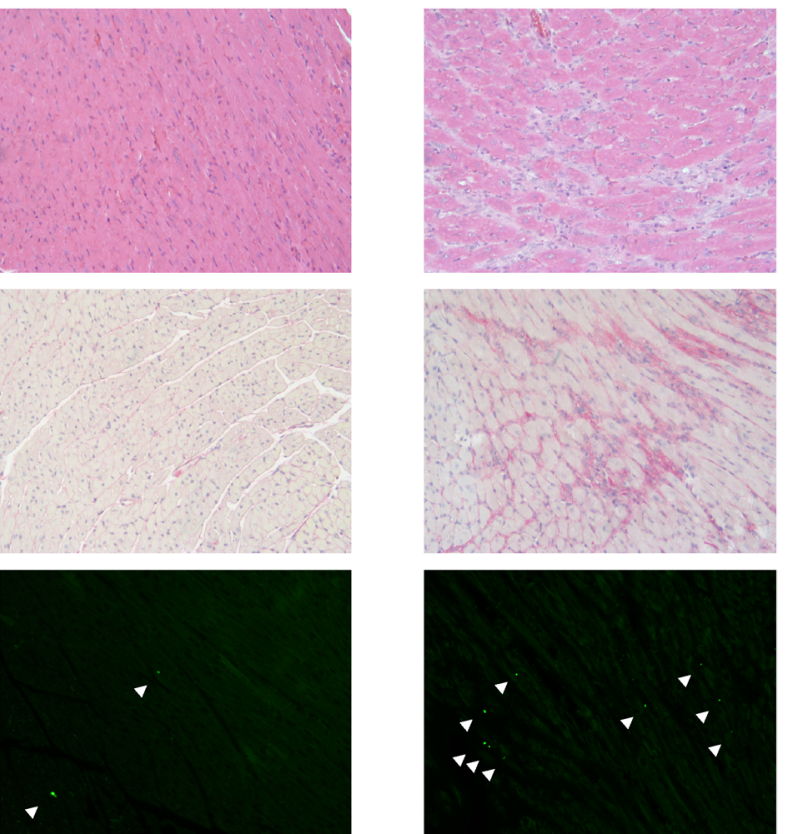

Figure 1. ISO-induced TTS-like cardiomyopathy in female WT mice. (A) Representative echocardiographic images showing apical LV ballooning. (B) Cardiac function in female mice $(n=5-10)$. (C) cTnT levels determined by ELISA. $n=8-10$. (D) Histological studies with myocardial sections. Fibrosis (picrosirius red staining) and TUNEL (FITC immunofluorescence) positivity was observed from the same regions with H\&E abnormality. Representative images are from 5 to 10 mice in each group. Scale bar: $50 \mu \mathrm{m}$. Dosing: ISO groups, $200 \mathrm{mg} / \mathrm{kg}$ ISO in PBS (i.p.); control groups: $100 \mu \mathrm{L}$ PBS (i.p.) as vehicle. (B and C) $P$ values are from 1-way ANOVA post hoc test with Tukey correction. d, days after injection.

affected cardiac macrophage populations. Using a previously established FACS protocol (21) (Supplemental Figure 2), we found that ISO induced a significant expansion of $\mathrm{CD}^{4} 5^{+} \mathrm{CD} 11 \mathrm{~b}^{+} \mathrm{F} 4 / 80^{+} \mathrm{Ly} 6 \mathrm{G}^{-}$cardiac macrophages in the acute TTS phase, which returned to basal levels after 7 days (Figure 2, A and B).

Because cardiac macrophages can increase through self-proliferation or monocyte infiltration (21), we further investigated the source of ISO-induced macrophages in the heart. Cardiac resident macrophages express Tim4, whereas monocyte-derived infiltrating macrophages do not (22). Therefore, we analyzed surface expression of Tim4 in the macrophage population to distinguish resident versus nonresident (infiltrating) macrophage subsets. At baseline, Tim $4^{+}$resident macrophages were the dominant subset $(21,22)$. In response to ISO stimulation, the cardiac macrophages subsets underwent a shift from Tim $4^{+}$resident macrophages to Tim4- infiltrating macrophages, suggesting that monocyte infiltration was the dominant mechanism for macrophage expansion in the ISO-stimulated heart (Figure 2, A and C). 
A

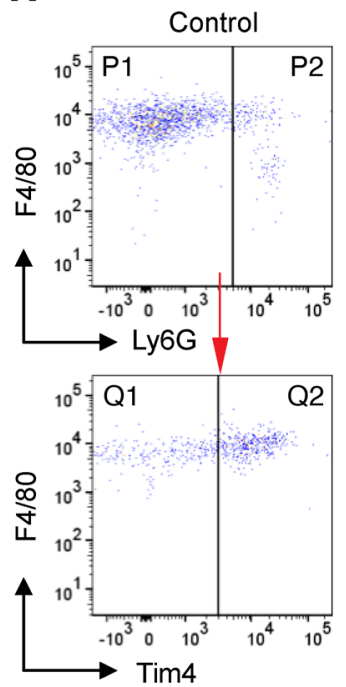

D

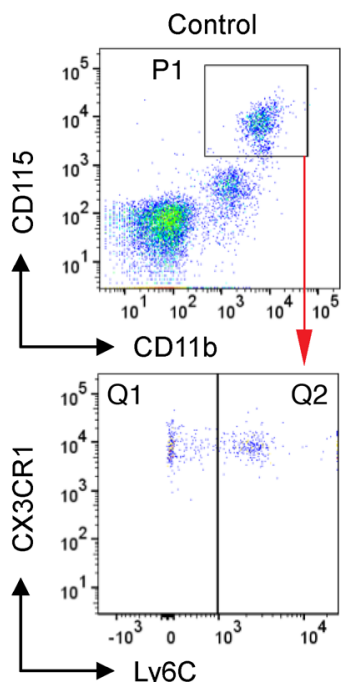

B

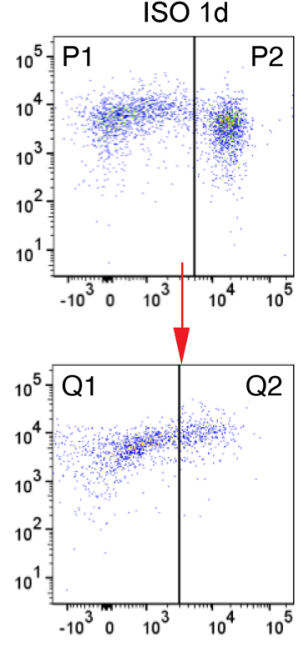

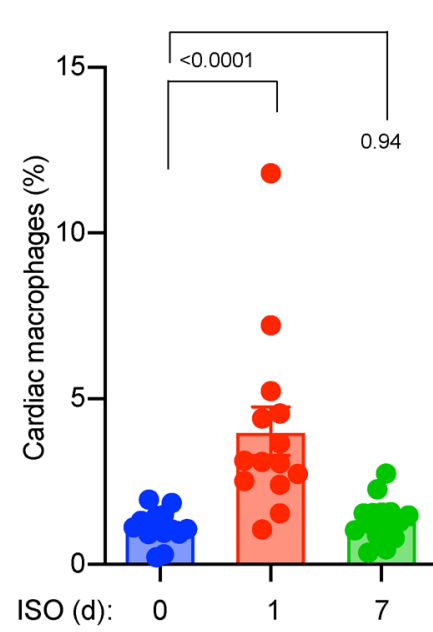

E

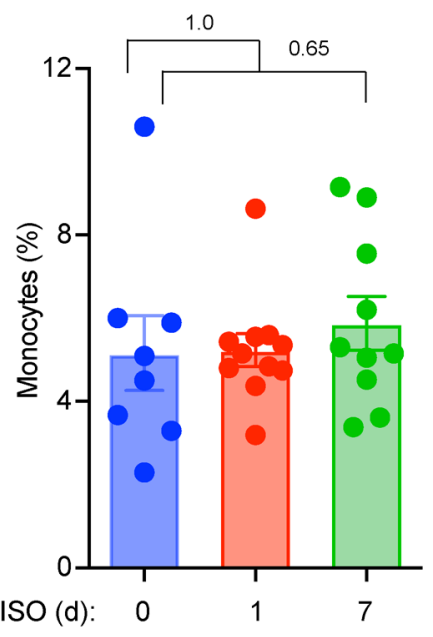

C

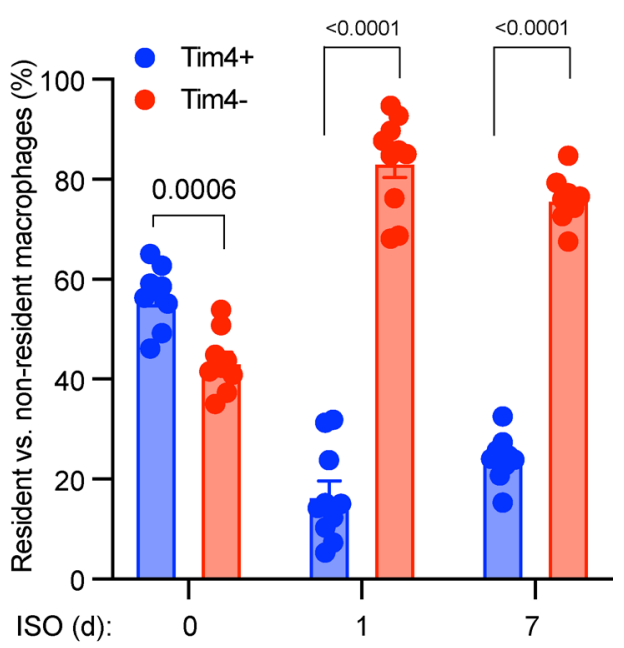

$\mathbf{F}$

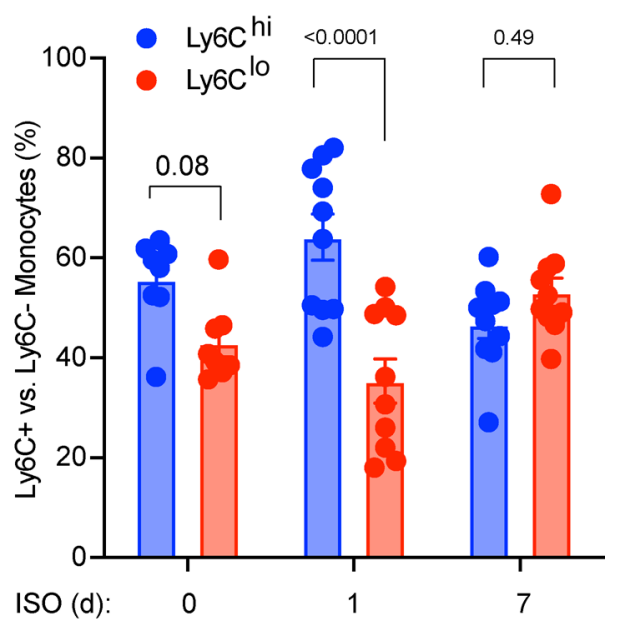

Figure 2. ISO-induced changes in cardiac macrophages and circulating monocytes. (A) Representative FACS plots of myocardial cells. P1, macrophages; P2, neutrophils; Q1, Tim4- infiltrating macrophages; Q2, Tim4+ cardiac resident macrophages. (B) Changes in cardiac cells after ISO treatment. The percentage of cardiac macrophages was calculated against all cells counted by flow cytometry, excluding debris. $n=14-16$. (C) The percentages of Tim4 ${ }^{+}$and Tim4- cardiac macrophage subsets (total cardiac macrophages, 100\%). $n=9-10$. (D) Representative FACS plots of peripheral blood monocytes. P1, monocytes (CD11b+CD115 hi); Q1, Ly6C ${ }^{10}$ monocytes; Q2, Ly6C hi monocytes. (E) Total monocytes in the blood at different days after ISO treatment (all white cells, 100\%). $n=8-11$. (F) The percentages of Ly6C ${ }^{\mathrm{hi}}$ and Ly6C ${ }^{\mathrm{lo}}$ monocytes (monocytes, $100 \%$ ). $n=8-10$. Dosing: ISO: 200 mg/kg. $P$ values were from 1-way ANOVA (B and E) and 2-way ANOVA (C and F) post hoc test with Tukey correction. d, days after ISO injection.

These data indicate that our model of ISO-induced mouse TTS-like cardiomyopathy recapitulated clinical TTS pathology characterized by cardiac macrophage infiltration and LV dysfunction.

Finally, we performed FACS studies with peripheral blood samples from ISO-treated mice and the control mice (Figure 2D). ISO did not trigger significant monocytosis (Figure 2E) but slightly shifted monocytes toward the proinflammatory Ly6 $\mathrm{C}^{\text {(hi) }}$ phenotype at day 1 after injection (Figure $2 \mathrm{~F}$ ). The mild changes in circulating monocyte numbers or activation status were in sharp contrast to the significant myocardial infiltration of macrophages (Figure 2C). We speculated that ISO did not directly activate monocytes to invade and injure the myocardium. Consistent with this view, we did not observe significant proinflammatory activation of bone marrow-derived macrophages (BMDMs) treated with ISO in vitro (Supplemental Figure 3). These observations suggest that the enhanced myocardial monocyte infiltration is unlikely consequent to direct ISO-mediated monocyte activation in the periphery but 
rather secondary to myocardial injury. These observations may also explain the clinical observations that beta blockers did not show benefits in TTS.

Dissecting the ISO-induced myocardial immune responses at the single-cell level. To gain additional insights into the myocardial immune response during ISO-induced TTS-like cardiomyopathy, we sorted CD45 cells from mouse hearts at 24 hours after injection (ISO vs. vehicle control), and performed single-cell RNA-Seq studies (scRNA-Seq).

After raw data processing and quality control, 5641 cells from the control group and 5437 cells from the ISO group were subjected to downstream bioinformatics analysis. Using Uniform Manifold Approximation and Projection (UMAP) for dimension reduction and unsupervised clustering analysis using Seurat pipeline, we identified 10 distinct cell populations from the total 11,078 cells (Figure 3A). Gene expression patterns of established canonical markers enabled us to assign putative biological identities to each cluster, namely macrophages, B cells, neutrophils, fibroblasts, DCs, T cells, NK cells, Th2 cells, endothelial cells, and mural cells (Figure 3B and Supplemental Figure 4).

Comparing the 2 groups (Figure 3, C and D), the macrophage cluster was the most abundant in the ISO group and increased approximately 2 -fold compared with the control group $(41.4 \%$ and $24.7 \%$, respectively), faithfully recapitulating the monocyte-macrophage infiltration phenotype (Figure $2 \mathrm{C}$ ). Other myeloid cells, such as neutrophils and DCs, were also increased in ISO group compared with the control group. In contrast, the lymphocyte clusters, such as B, T, Th2, and NK cells, were all reduced in the ISO group. The endothelial and mural clusters comprised a negligible percentage of cells $(<0.5 \%)$ and were excluded from downstream analyses.

Because the macrophage was the most abundant cluster, we further sub-clustered it by K-nearest neighbor, graph-based, unsupervised clustering (the FindNeighbors function) and identified 2 macrophage subsets (clusters 0 and 1) that showed drastic differences between the ISO and control groups (Supplemental Figure 5A and Figure 3E). Upon ISO stimulation, there was approximately a 2-fold increase in cluster 0 and a 3-fold reduction in cluster 1 (Figure 3F). Gene expression patterns of known macrophage markers identified cluster 0 as the CCR2 ${ }^{+}$infiltrating macrophages and cluster 1 as the Tim $4^{+}$Lyve $1^{+}$resident macrophages (Figure $3 \mathrm{G}$ and Supplemental Figure 5, B and C). In addition, the infiltrating macrophages (cluster 0 ) expressed high levels of classic inflammatory (M1) genes such as CD14 and IL1 $\beta$ (Supplemental Figure 5D), whereas the resident macrophages (cluster 1) expressed M2 markers such as CD163 and FOLR2 (Supplemental Figure 5E). Common macrophage genes, such as ITGAM (Mac-1), EMR1 (F4/80), and FCGR1 (CD64) were expressed in both subsets (Figure 3G and Supplemental Figure 5F). As previously reported (19), MHC-II genes (H1-Aa and H2-Ab1) were also similarly expressed in both macrophage subsets (Supplemental Figure 5G). Collectively, these clustering results are consistent with the FACS data that show ISO-induced myocardial infiltration of monocytes and monocytes further differentiated into CCR2 ${ }^{+}$Tim4 $4^{-}$inflammatory macrophages in the myocardium.

Next, we performed gene ontology (GO) analysis with differentially expressed genes (DEGs; ISO vs. control, adjusted $P<0.05$; Supplemental Figure 6). DEGS were analyzed in 2 groups: (a) genes upregulated in ISO group, and (b) genes upregulated in control group (downregulated in the TTS group). As shown in Figure 4, DEGs upregulated in the myeloid cells (i.e., macrophages, neutrophils, and DCs) from ISO group showed similar enrichment in GO terms related to energy metabolism (adenosine triphosphate biosynthesis and metabolic processes), metabolism of purine ribonucleotides and purine-containing compounds, ribose phosphate and nucleoside monophosphate metabolic processes, and ribonucleoprotein complex-related processes, indicating a robust activation status of the myeloid cells in TTS myocardium. Furthermore, purine ribonucleotides and purine-containing compounds are classic regulators of macrophage inflammation $(24,25)$. In contrast, DEGs upregulated in control myeloid cells (downregulated in the ISO group) were enriched in GO terms related to classic innate immune functions and were more diverse among cell types (Supplemental Figure 7). Similar GO patterns were observed in lymphocyte clusters (Figure 4 and Supplemental Figure 7). The overlapping GO terms in ISO lymphocytes were closely associated with inflammation, indicating activation of lymphocytes. The GO terms enriched in control lymphocytes were different for each cell type, concordant to their distinct functions at steady state. However, the significant reduction of lymphocyte numbers in ISO-treated mouse hearts indicates they might not be the primary regulator of TTS-like pathogenesis (Figure 3D).

Depletion of macrophages and monocytes ameliorates ISO-induced TTS-like cardiomyopathy. The significant abundance of cardiac macrophages and their increase after ISO treatment suggest these cells may play a 
A

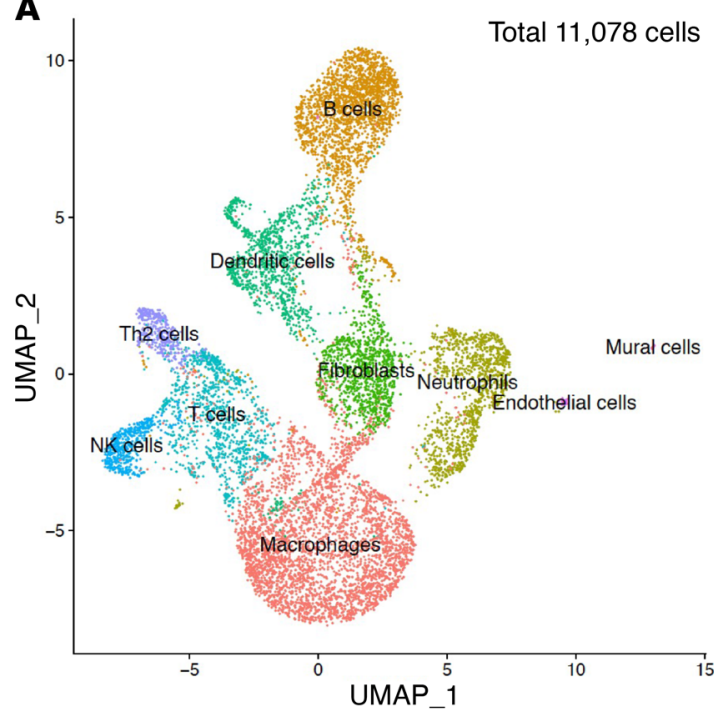

C

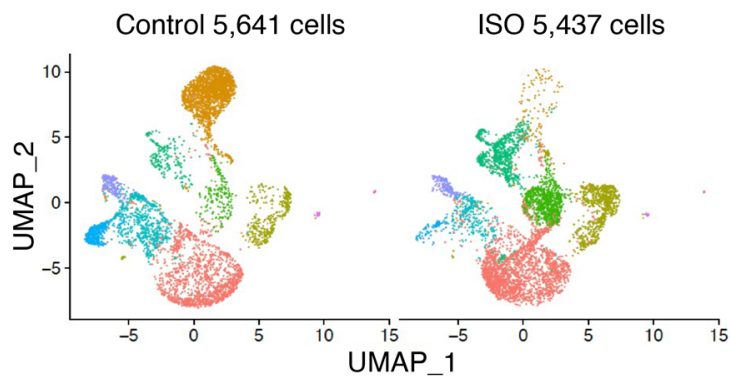

E

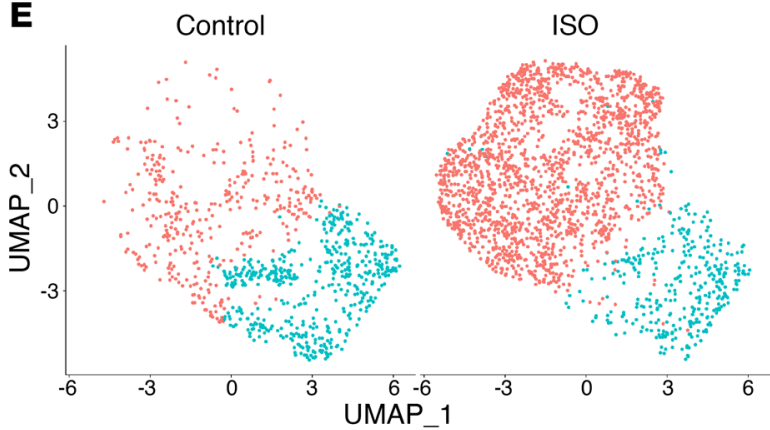

G

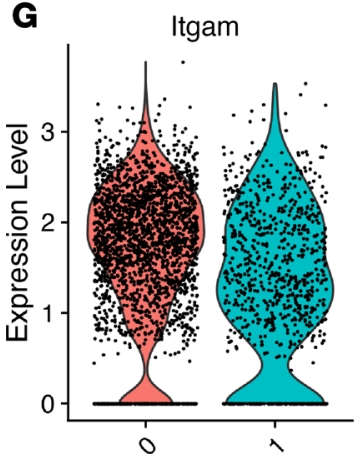

B

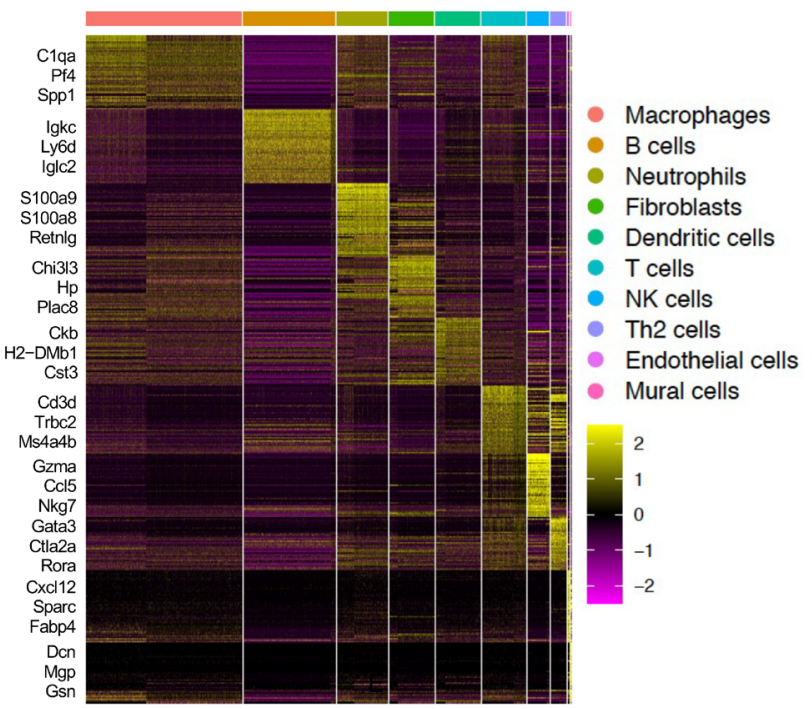

D

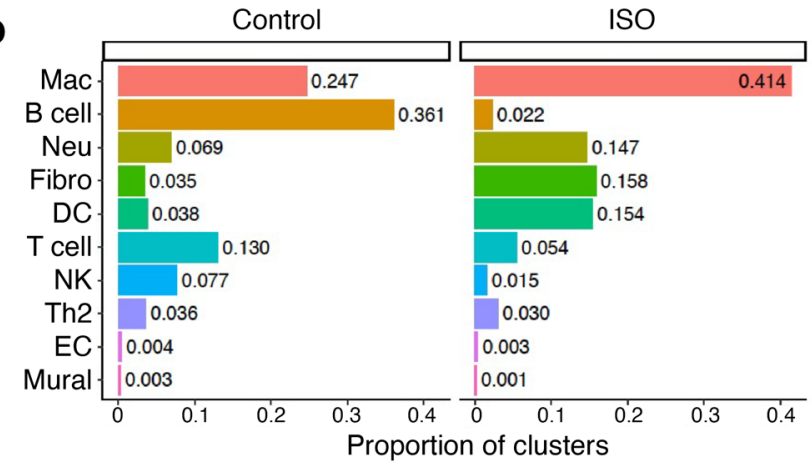

$\mathbf{F}$



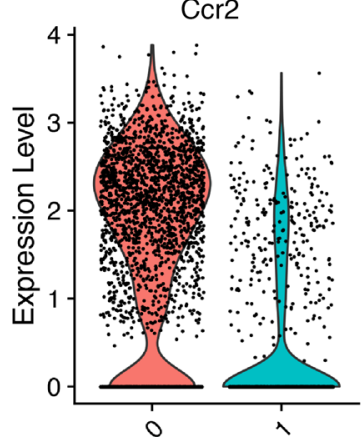

Lyve1


Figure 3. scRNA-Seq study on ISO-induced myocardial immune responses. (A) Ten distinct cell clusters were identified from the total of 11,078 cells. (B) Heatmap of the top 50 marker genes for each cluster. Selected cell type-specific markers are labeled. (C) UMAP of control and TTS cells. (D) Proportion of cell clusters before and after ISO administration. (E) Two subsets were identified (designated 0 and 1) from cardiac macrophages (Mac). (F) Proportion of Mac subclusters before and after ISO. (G) Violin plots showing expression of Mac marker genes in 2 subclusters. Three mouse hearts were pooled in each group $(n=3)$. Ccr2, a marker of cardiac infiltrating Macs; EC, endothelial cell; Fibro, fibroblast; Itgam, also known as Mac-1, a common marker of macrophages; Lyve1, marker of cardiac resident macrophages; Neu, neutrophil; Tim4, marker of cardiac resident macrophages. 


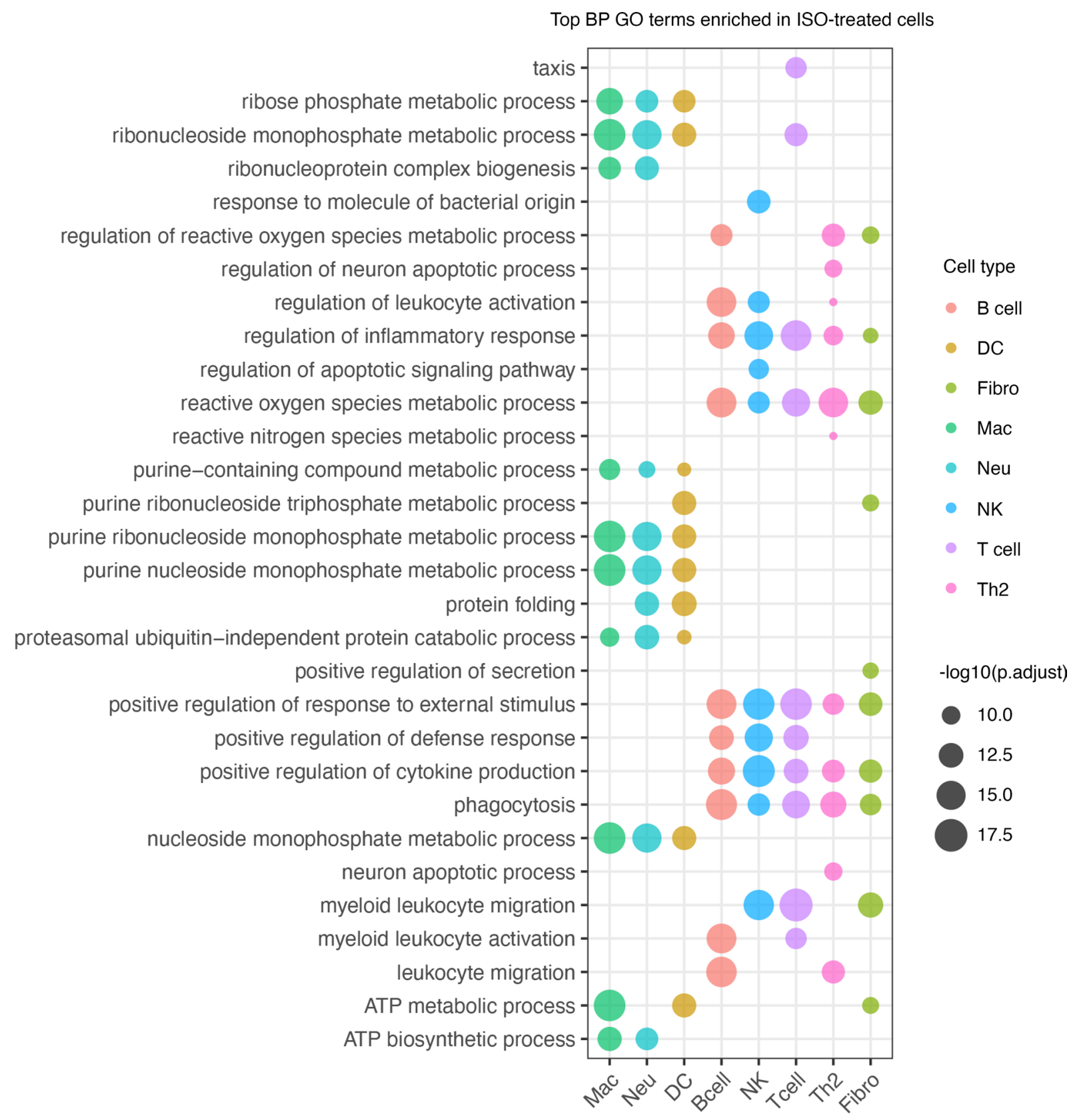

Figure 4. ISO-induced GO pathway enrichment in myocardial immune cells. GO analyses with DEGs that were upregulated in the ISO-treated group (TTS). Top 10 biological process (BP) GO terms according to adjusted $P$ values (p.adjust) are shown. Fibro, fibroblast; Mac, macrophage; Neu, neutrophil.

pathogenic role in TTS-like cardiomyopathy. Therefore, we sought to target these cells to ameliorate TTS. We and others have shown that administration of clodronate-containing liposomes significantly depletes both circulating monocytes and tissue macrophages in vivo $(21,26)$. We found that mice pretreated with clodronate-containing liposomes (depleted of macrophages and monocytes) exhibited profound protective resistance to ISO-induced cardiac dysfunction (Figure 5A), suggesting a pathogenic role of macrophages in TTS-like cardiomyopathy.

To establish a role of monocyte infiltration, we studied TTS-like phenotypes in CCR2-KO mice, which lack circulating monocytes and are defective in chemotaxis (21). Compared with WT controls, CCR2-KO mice had significantly preserved LVEF after ISO injection (Figure 5B), conversely indicating a pathogenic role of monocytes in TTS-like cardiomyopathy. Next, we treated WT mice with RS-504393 (RS), a small molecular CCR2 antagonist (21), and found that RS administration significantly improved LVEF (Figure 5C). 
Accordingly, the cardioprotective effects of RS were likely due to the blockade of myeloid cell infiltration, as revealed by FACS studies (Figure 5D). Collectively, these data not only suggest a causative role of monocytes and macrophages in pathogenesis of TTS-like cardiomyopathy but suggest that targeting these cells maybe therapeutically efficacious.

Genetic damping of myeloid activation ameliorates ISO-induced TTS-like cardiomyopathy. It is well known that Hif1 $\alpha$ is a critical transcription factor in myeloid cell activation (27). As such, we used Lyz2-Cre-driven myeloid Hif1 $\alpha$-deficient mice (Hif1 $\alpha-\mathrm{KO})$ as a myeloid loss-of-function model and studied ISO-induced TTS-like cardiomyopathy. In response to ISO surge, the Hif1 $\alpha$-KO hearts accumulated significantly fewer macrophages and neutrophils (Figure 6, A-C), suggesting a crucial role of Hif1 signaling in TTS-associated myeloid activation. Consistently, Hif1 $\alpha-\mathrm{KO}$ mice also exhibited significant resistance to ISO-induced TTS-like cardiomyopathy, showing reduced plasma cTnT levels (Figure 6D) and preserved LVEF (Figure $6 \mathrm{E})$. Collectively, these data demonstrate that Hif1 signaling is requisite to support myeloid activation in ISO-induced TTS-like cardiomyopathy.

Bortezomib inhibits myeloid activation in ISO-induced TTS-like cardiomyopathy: an immunomodulatory therapy. Given the critical role of monocytes and macrophages in ISO-induced TTS-like cardiomyopathy, we hypothesized that suppressing myeloid activation could favorably affect heart function. We tested this hypothesis with bortezomib (BZ), an FDA-approved drug. BZ was originally designed as a proteasome inhibitor that suppresses cancer cell proliferation via inhibiting proteasome-mediated degradation of tumor-suppressor proteins. We and others have shown that BZ, at very low nontumor-suppressive doses, can modulate immune cell activation $(28,29)$. Recent clinical studies demonstrated that BZ is an effective immunomodulatory therapy for arthritis, transplant rejection, graft-versus-host disease, and autoimmune diseases (30-34). BZ appears to be a safe immunomodulatory drug with a very favorable side effect profile (35). Here, we assessed if immunomodulatory therapy using BZ could ameliorate TTS in mice.

We first performed FACS analysis with ISO-treated and control mouse hearts and found that BZ administration significantly blocked myocardial infiltration of macrophages and neutrophils (Figure 7 , A-C). Furthermore, echocardiography revealed improved LVEF in BZ-treated mice compared with the vehicle-treated (PBS) group at 1 day after ISO administration (Figure 7D). ISO-induced cardiac cell death and fibrosis by day 7 was also attenuated by BZ administration (Figure 7, E and F). Therefore, immunomodulatory therapy with BZ attenuated myeloid activation and ameliorated TTS-like pathogenesis.

Using in vitro cell culture models, we further studied the effects of BZ on cardiomyocytes and macrophages, respectively. BZ did not exhibit cardiotoxicity but had marginal hypertrophic effects on cardiomyocytes (Figure 7G). In contrast, BZ exhibited strong antiinflammatory effects in macrophages. Using LPS as a mimic of pathogen-associated molecular patterns and damage-associated molecular patterns, we treated BMDMs in vitro to trigger proinflammatory activation of macrophages. BZ significantly inhibited LPS-induced expression of proinflammatory genes, including TNF- $\alpha$ (Tnfa), IL1 $\beta$ (Il1b), CCL2 (Ccl2), Cox2 (Ptgs2), CD64, and Nlrp3 (Figure 7H and Supplemental Figure 8). Taken together, these data strongly support an immunomodulatory and myeloid inhibitory role of BZ in ISO-induced TTS-like cardiomyopathy.

\section{Discussion}

TTS is a stress-induced cardiomyopathy without an effective therapy because of its unknown pathogenic mechanisms. Using a mouse model of ISO-induced TTS-like cardiomyopathy, we identified cardiac-infiltrating macrophages as critical to TTS pathogenesis. This conclusion is supported by the observation that genetic or pharmacologic approaches that prevent macrophage infiltration or activation ameliorate ISO-induced cardiac dysfunction and tissue injury. Our findings also suggest that FDA-approved immunomodulatory therapies (e.g., BZ) may be effective in reducing cardiac dysfunction and accelerating recovery.

An essential aspect of our work relates to identifying monocytes and monocyte-derived cardiac-infiltrating macrophages as critical regulators of TTS that can be therapeutically targeted. Hearts have 2 major subsets of macrophages: yolk sac-derived resident macrophages and monocyte-derived nonresident, infiltrating macrophages. Recently, studies by our group and others have revealed the distinct roles of monocytes and macrophages in the heart during acute and chronic stresses $(20,21,36)$. Monocytes and monocyte-derived macrophages appear to have a proinflammatory detrimental role in the heart. In contrast, the cardiac resident macrophages appear to be antiinflammatory and cardioprotective. Clinical TTS was associated with myocardial accumulation of macrophages $(15,16)$, and we found the same phenotype in ISO-treated mice. 
A

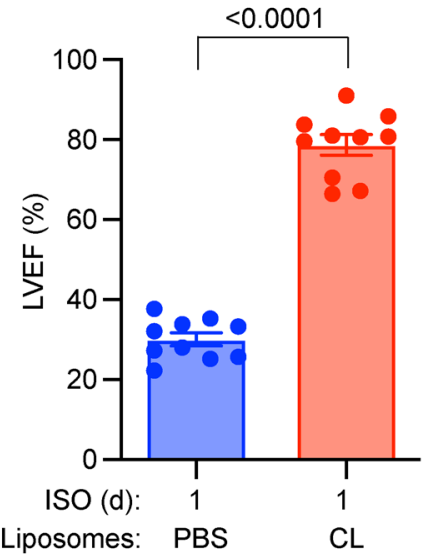

B

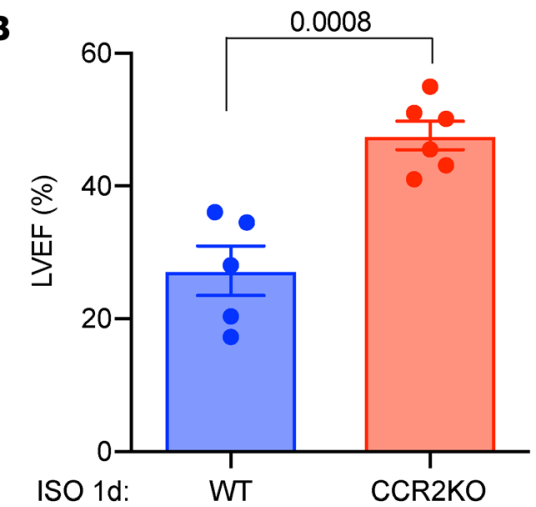

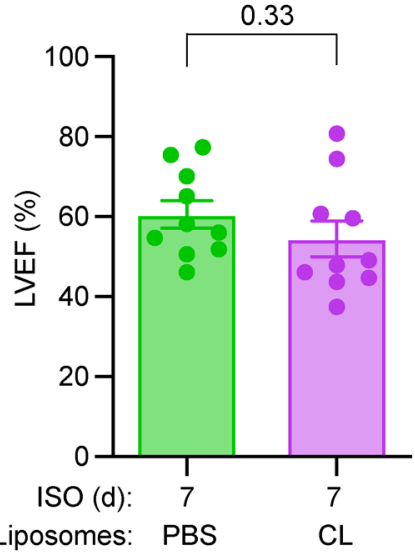

C



D


E


Figure 5. Depletion of macrophages and monocytes ameliorates TTS-like cardiomyopathy in mice. (A) Cardiac function in mice pretreated with clodronate-liposomes (CL) or PBS-liposome (PBS). $n=10$. (B) Cardiac function in CCR2-KO and WT mice. $n=5$. (C) Cardiac function in WT mice pretreated with RS-504393 (RS; $2 \mathrm{mg} / \mathrm{kg}$ twice daily by oral gavage) or PBS (Veh). $n=$ 14. (D) Representative FACS plots of myocardial cells. Mice were pretreated with CL or RS for 3 days before ISO injection. P1, macrophages; P2, neutrophils. (E and $\mathbf{F}$ ) Changes in cardiac macrophages $(\mathbf{E})$ and neutrophils $(\mathbf{F}) . n=5-10$. (A-C) $P$ value from 2-tailed unpaired Student $t$ test. (E and F) $P$ values were from a 1-way ANOVA post hoc test with Tukey correction. 
A

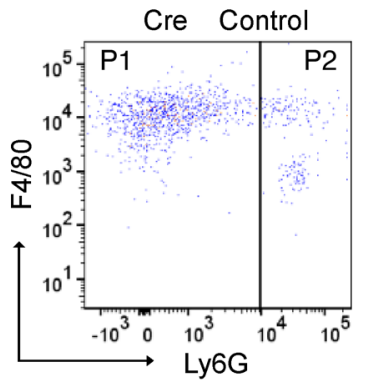

B

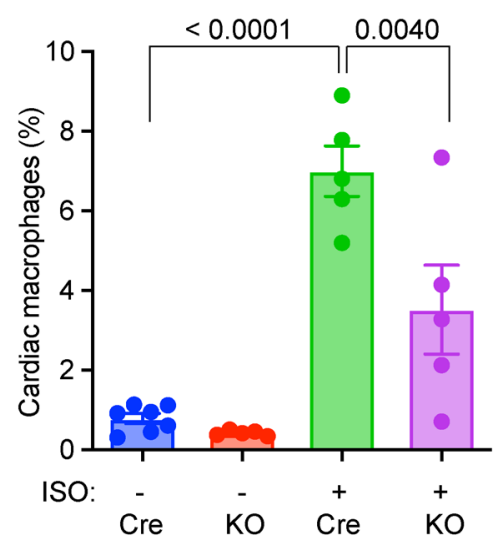

D

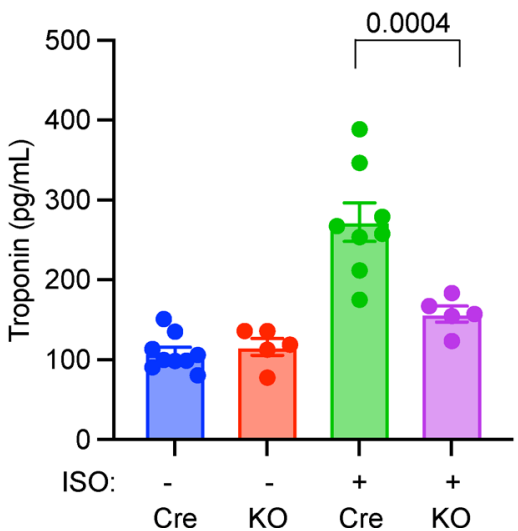

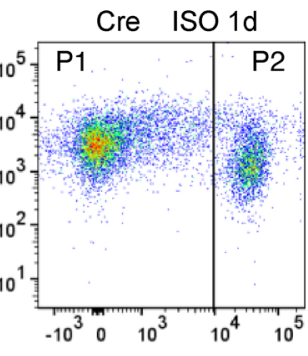

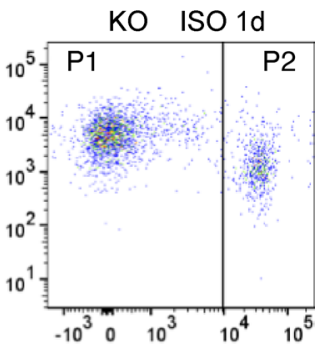

C

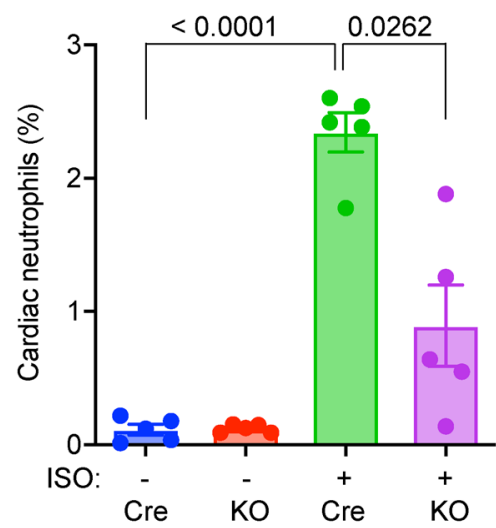

E

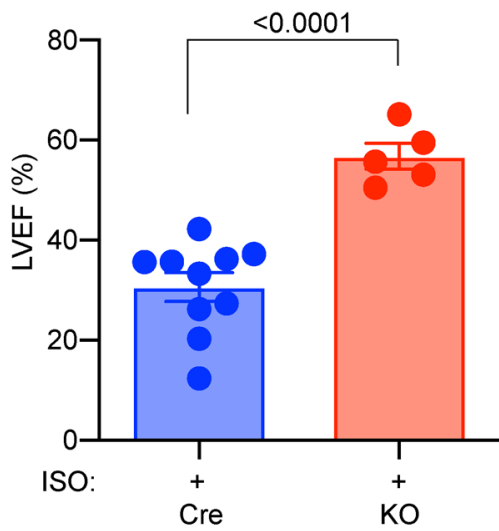

Figure 6. Genetic model of myeloid inhibition ameliorates ISO-induced TTS-like cardiomyopathy. (A) Representative FACS plots of myocardial cells from Lyz2-Cre (Cre) and Lyz2-Hif1 $\alpha$-KO (KO) mice. P1, macrophages; P2, neutrophils. (B and C) Changes in cardiac macrophages (B) and neutrophils (C). $n=5-7$. (D) Plasma cTnT levels. $n=5-9$. (E) Cardiac function in ISO-treated Cre and KO mice. $n=5-10$. All studies were performed at day 1 after ISO. (B-D) $P$ values are from a 2-way ANOVA post hoc test with Tukey correction. (E) $P$ value is from 2-tailed unpaired Student $t$ test.

Based on the expression of multiple macrophage subset markers, such as TIM4, LYVE1, and CCR2, data from FACS and scRNA-Seq studies strongly supported ISO-induced monocyte infiltration into the myocardium, which gave rise to CCR $2^{+}$nonresident macrophages. These cells likely mediated myocardial proinflammatory responses, as indicated by ISO-induced enrichment in inflammation-related GO terms. Accordingly, blockade of monocyte infiltration or proinflammatory activation protected the heart from ISO-induced cardiac dysfunction. As such, these data identify monocytes and monocyte-derived infiltrating macrophages as critical regulators of TTS pathogenesis and potential therapeutic targets. However, we have not confirmed a role of cardiac resident macrophages in TTS. Given that the Tim $4^{+} \mathrm{Lyve} 1^{+}$cardiac resident macrophages were significantly reduced after ISO challenge, it is plausible that the loss of these cells may contribute to cardiac dysfunction. Studies with inducible depletion or mutation of resident macrophages (i.e., using a $\mathrm{Cx} 3 \mathrm{cr} 1$-based system, ref. 22) would be very interesting to explore the functions of cardiac resident macrophages in TTS. 
A
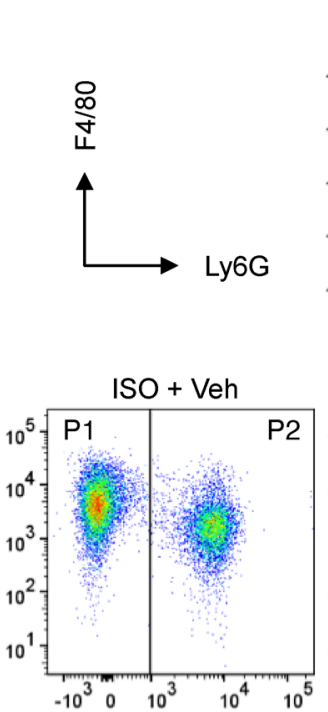

D

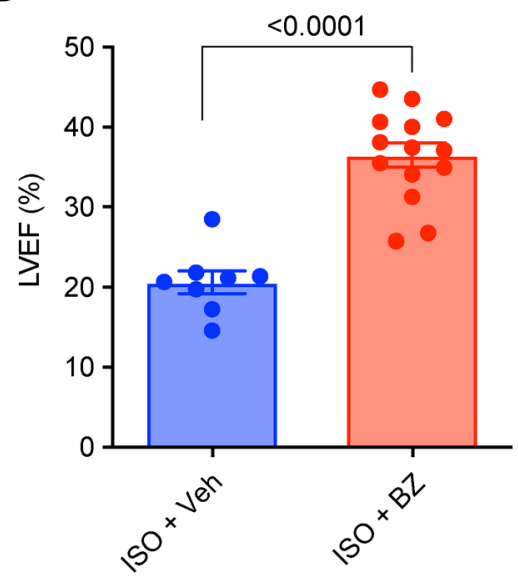

G



B
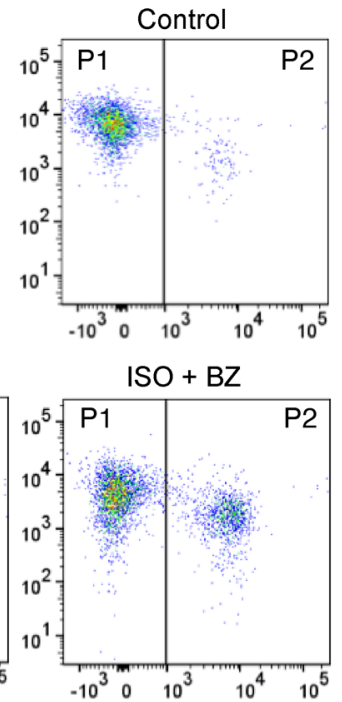

E

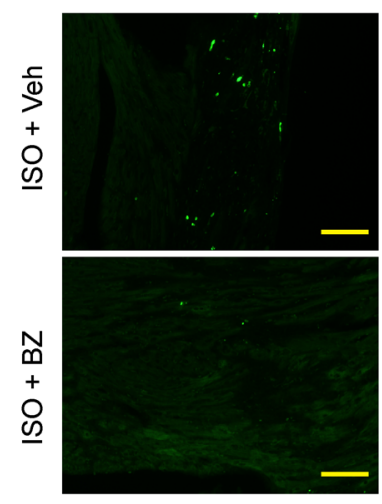

TUNEL
C

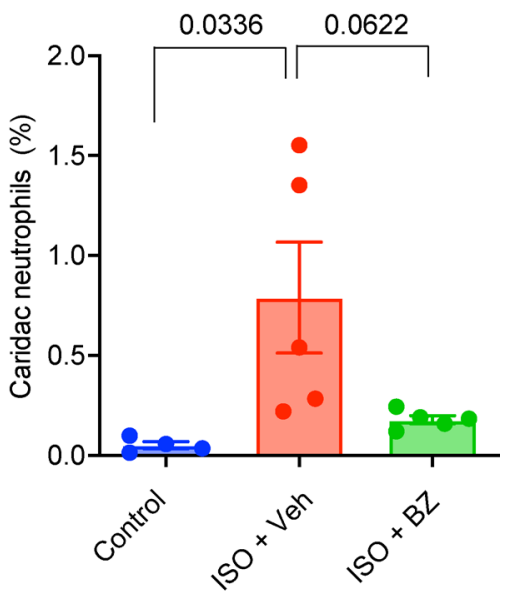

$\mathbf{F}$

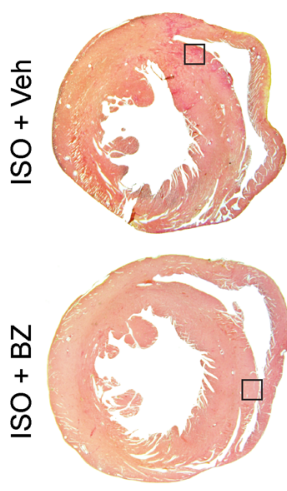

PicroSirius Red

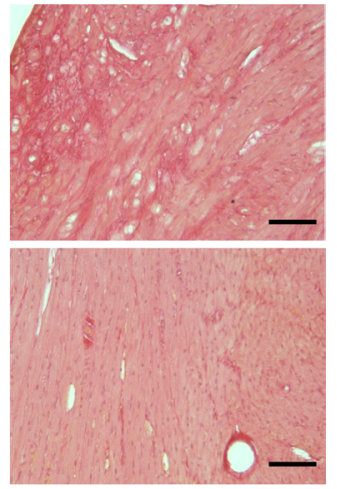

H
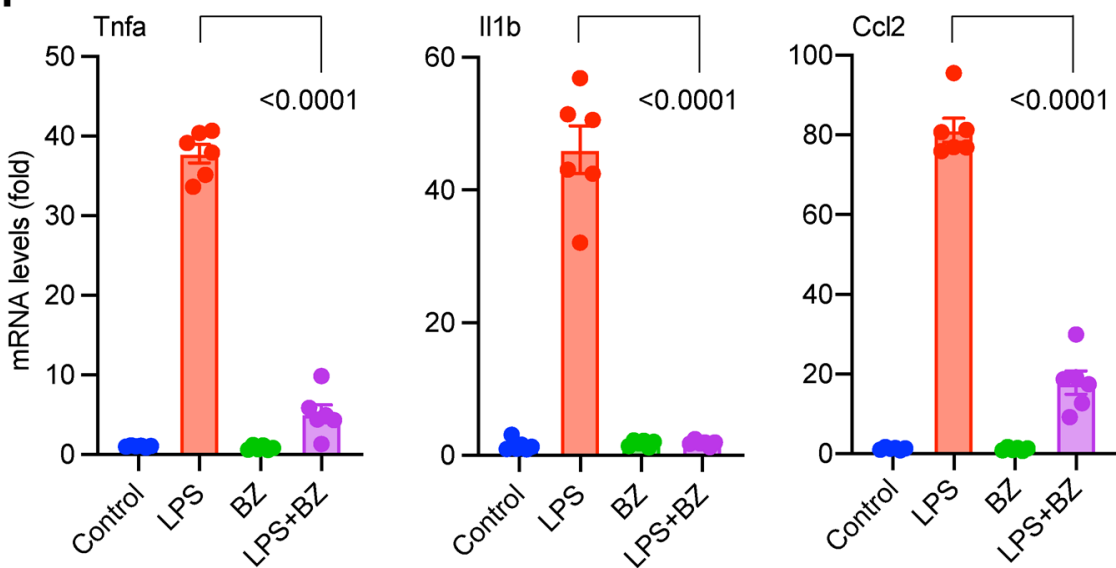

Figure 7. Pharmacological inhibition of myeloid activation ameliorates ISO-induced TTS-like cardiomyopathy. (A) Representative FACS plots of myocardial cells. P1, macrophages; P2, neutrophils. (B and C) Changes in cardiac macrophages (B) and neutrophils (C). $n=4-7$. (D) Cardiac function in ISO-treated mice with or without BZ therapy. $n=8-14$. (E) Cell death in the myocardium detected by TUNEL staining. (F) Myocardial fibrosis detected by picrosirius red staining. Boxed areas shown in high magnification on the right. Representative images are from 5 to 10 mice in each group. Scale bar: $50 \mu \mathrm{m}$. (G and $\mathbf{H}$ ) Effects of BZ on cardiomyocytes (G) and macrophages $(\mathbf{H})$ in vitro. $n=6$ in each group. BZ dose, $5 \mu$ M, added 6 hours before phenylephrine (PE; $50 \mu \mathrm{M}$, treated for 24 hours) or LPS ( $50 \mathrm{ng} / \mathrm{mL}$; treated for 24 hours). (A-D) One day after ISO administration. (E and F) Seven days after ISO administration. BZ treatment started 3 days before ISO administration ( $0.3 \mathrm{mg} / \mathrm{kg}$ i.p. every either day). (D) $P$ values were from 2 -tailed unpaired Student $t$ test. (B and $\mathbf{C}$ ) $P$ values were from a 1-way ANOVA post hoc test with Tukey correction. (G and $\mathbf{H}) P$ values were from a 2 -way ANOVA post hoc test with Tukey correction. 
In addition, ISO also induced expansions of neutrophils and DCs in the myocardium, despite there being fewer of these cells than macrophages. The GO terms enriched in these myeloid cells indicated they might have similar proinflammatory roles as the macrophages. More investigation is warranted of these myeloid cells. Contrary to the innate immune cells, lymphocytes were reduced in response to acute ISO challenge, indicting they likely do not drive TTS onset. Given the similar pattern in ISO-induced GO enrichment in lymphocytes, these cells appeared to be activated and might participate in cardiac remodeling after TTS. The functions of the adaptive immune system in TTS remain to be explored.

One of the limitations of this study is that we cannot precisely define a molecular regulator(s) of TTS pathogenesis. The scRNA-Seq data demonstrated complex immune responses in the ISO-treated myocardium, involving almost all immune cell types and forming a vast signaling network. The efforts to identify a specific cytokine or pathway have not been successful. However, as a complex syndrome, TTS pathogenesis may, indeed, be multifactorial. We speculate that TTS pathogenesis is an interplay between stress-injured myocardium and the immune system that responds to injury. Our data do not support ISO-induced direct activation of monocytes but suggest a response-to-injury model. The SNS signals to immune cells mainly through $\beta 2 \mathrm{AR}$ to mediate antiinflammatory or immunosuppressive functions (37), contradicting the proinflammatory TTS phenotype. Moreover, if myeloid cells are not activated by catecholamines, it is understandable that beta blockers cannot benefit clinical TTS because, at the point of TTS diagnosis, myocardial injury has been done and myeloid cells have been activated. The true upstream activators and downstream effectors of myeloid cells remain to be investigated and the scRNASeq data set would be a foundation of future studies.

Another limitation of this study is that the ISO-induced mouse cardiomyopathy model may be too simplified compared with clinical TTS, a unique and enigmatic cardiomyopathy triggered by extreme emotional or physical stress. Clearly, there is no way to model the human emotional stress in mice and the physical stress cannot be as simple as ISO surge. However, we think this mouse TTS model is practical and useful in TTS-related research. Our data are significant at 3 levels: they (1) recapitulated the transient cardiac dysfunction phenotype; (2) were associated with myocardial infiltration of macrophages; and (3) effectively worked for therapeutic models.

In a recent article, Forte et al. (38) reported that high-dose ISO induced type 2 myocardial infarction in mice, in which cardiac inflammation and fibrosis developed 2 to 8 weeks after injection. Their finding is consistent with our 1 -week ISO data, likely reflecting the long-term cardiac remodeling. Instead, in the present study, we mainly focused on the acute (i.e., 1-day) TTS-like responses and showed severe cardiac dysfunction (LVEF $<40 \%$ ) that recovered significantly within 7 to 10 days (baseline vs. day 7 LVEF: $70 \%$ vs. $60 \%$ ), similar to transient TTS. Therefore, these 2 studies share certain similarities in terms of long-term cardiac remodeling but significantly differ in the subject of investigation. Furthermore, our data demonstrated that cardiac macrophage infiltration preceded myocardial cell death and fibrosis, indicating a pathogenic role of macrophages. Finally, pharmacologically targeting monocytes and macrophages profoundly ameliorated ISO-induced TTS-like cardiomyopathy, not only confirming the causative roles of these immune cells in TTS pathogenesis but also providing targets for development of novel TTS therapies.

\section{Methods}

Supplemental figures and tables can be found online. The scRNA-Seq data are deposited in the Gene Expression Omnibus (accession no. GSE189358).

\section{Animals}

All mice were on a C57BL/6J background. Mice with a myeloid-specific deficiency of Hif1 $\alpha$ (Lyz2-Cre: Hif1 $\alpha^{\mathrm{f} / \mathrm{fl}}$ [designated Hif1 $\alpha$-KO]) were described previously (39). The Lyz2-Cre line was used as a genetic control. WT C57BL/6J mice (WT) were purchased from The Jackson Laboratory. Mice were housed in a temperature- and humidity-controlled, specific pathogen-free facility with a 12-hour light/dark cycle and ad libitum access to water and chow.

\section{Cell culture}

Mouse BMDMs were differentiated in DMEM containing 10\% FBS and 25\% L-929 conditioned medium as described previously (40). Treatment was performed in DMEM containing $10 \%$ FBS. 
Neonatal rat ventricular myocytes were isolated from day-2 Sprague-Dawley rat pups and cultured as described previously (41). Treatment was performed in serum-free DMEM containing 1\% insulin-transferrin-sodium selenite media supplement (Sigma-Aldrich, I1884).

\section{Mouse TTS model}

Administration of a single high dose of ISO causes TTS-like responses in rodent models $(42,43)$. Female and male mice at 8 to 16 weeks of age received a single-dose i.p. injection of ISO ([-]-isoproterenol hydrochloride, Sigma-Aldrich, I6504) dissolved in $100 \mu \mathrm{L}$ sterile Dulbecco's PBS (Thermo Fisher Scientific, SH30028.03). The control group received an i.p. injection of $100 \mu \mathrm{L}$ PBS as vehicle. The ISO dose was titrated between 100 and $500 \mathrm{mg} / \mathrm{kg}$ and fixed at $200 \mathrm{mg} / \mathrm{kg}$ for female mice. Cardiac function was monitored by transthoracic echocardiography before and after ISO injection for up to 10 days. For echocardiography, mice were anesthetized by inhalation of $0.1 \%$ to $0.5 \%$ isoflurane vaporized in $100 \%$ oxygen, keeping a steady heart rate above $500 \mathrm{bpm}$; LV recording was obtained in the parasternal long-axis view in B-mode on a Vevo-770 or 3100 System (Fujifilm) (41). LVEF was calculated as the percentage of LV fractional area change, using the VevoLab software. Plasma levels of cTnT were determined by a Mouse Cardiac Troponin T ELISA Kit (MyBioSource Inc., MBS726068).

\section{Flow cytometry}

The mouse heart was perfused, excised, minced, digested with type I collagenase, mechanically disrupted, and filtered through a $70 \mu \mathrm{m}$ cell strainer to get a single-cell suspension (21). Cells were collected by centrifugation, subjected to live-dead dye (LIVE/DEAD Fixable Yellow Dead Cell Stain Kit, Invitrogen, L34968) and surface antibody staining (fluorescent conjugated primary antibodies; BioLegend), and analyzed by flow cytometry. Cells were also sorted by flow cytometry to collect live CD45 ${ }^{+}$cells for transcriptomic studies. The following fluorescent antibodies were used in this study: CD45-PerCP (BD Biosciences, 561047), CD11b-AF488 (BD Biosciences, 557672), Ly6G-APC-Cy7 (BD Biosciences, 560600), F4/80-BV421 (BioLegend, 123137), and CD45-FITC (BioLegend, 103108).

\section{Histology}

Cardiac tissue samples were fixed in 10\% neutralized formalin and embedded with paraffin following standard protocols. H\&E staining was perform using Mayer's hematoxylin solution and eosin Y solution (Sigma-Aldrich, 51275, 318906). Fibrosis was stained using the Picrosirius Red Stain Kit (Polysciences, 24901) (41). TUNEL staining was performed with the ApopTag Fluorescent In Situ Apoptosis Detection Kit (Millipore, S7110) (44). Microscopic images were analyzed using NIH ImageJ software.

\section{RNA extraction and quantitative real-time PCR}

Total RNA was purified using the Qiagen RNeasy Mini Kit, following the manufacturer's protocol. RNA was reverse transcribed to complementary DNA using the iScript Reverse Transcription Kit (Bio-Rad, 170-8841). Quantitative real-time PCR was performed with the TaqMan method using the Roche Universal ProbeLibrary as TaqMan probes. Relative expression was calculated using the $\Delta \Delta \mathrm{Ct}$ method with normalization to Gapdh.

\section{Single-cell RNA-Seq studies}

Single-cell library construction and sequencing. $\mathrm{CD} 45^{+}$cells were sorted from mouse myocardium by flow cytometry. Dead cells were excluded by the live-dead dye. A total of $15,000 \mathrm{CD} 45^{+}$cells were input to the 10x Genomics Chromium Controller for single-cell partitioning and Gel Bead-in-Emulsion (GEM) generation. GEMs are reaction vessels in which cDNA from each single cell is generated and uniquely barcoded. cDNA libraries were checked for quality using an Agilent 2100 Bioanalyzer. Adapters were ligated, and barcoding was accomplished for each library, followed by an additional assessment of average fragment size using the bioanalyzer. Samples were pooled and sequenced on a Novaseq 6000 S4 flow cell using the sequencing parameters outlined in the $10 \times$ Genomics Chromium Controller protocol (version CG000204).

Single-cell RNA-Seq analysis. Raw sequencing data in FASTQ format were processed with UMI-tools (version 1.0.0) to generate a white list for cell barcodes and unique molecular identifiers and to extract valid sequencing reads, aligned to the mouse reference genome ( $\mathrm{mm} 10$ ) with STAR (version 2.7.3a), and quantified with Subread featureCounts (version 1.6.4). Data normalization, cell clustering, and visualization were performed using Seurat (version 3.1.5). For initial quality control of the extracted 
gene-cell matrices, we filtered cells using the following parameters: low.threshold $=500$, high.threshold $=6000$ for number of genes per cell (nFeature_RNA); high .threshold $=25 \%$ for percentage of mitochondrial genes (percent.mito); and genes with parameter min.cell $=3$. Filtered matrices were normalized by the LogNormalize method with a scale factor of 10,000 .

Variable genes were found with parameters of selection.method $=$ vst and nfeatures $=2000$, trimmed for the genes related to cell cycle (GO:0007049), and then used for data integration (IntegrateData with FindIntegrationAnchors function), data scaling (ScaleData), and principal component analysis (RunPCA). Statistically significant principal components were determined by the JackStraw method, and the first 9 principle components were used for nonlinear dimensional reduction (UMAP) and clustering analysis (FindNeighbors) with resolution of 0.1 .

GO terms enrichment analysis. DEGs for each cluster between Cre and K2-KO samples were identified using the FindAllMarkers function of the Seurat package with options of only.pos $=$ TRUE, min.pct $=0.25$, and logfc.threshold $=0$. Enriched GOs of molecular function, biological function, and cellular component were identified using the enrichGO function of the clusterProfiler R package with a $q$ value cutoff of 0.1 and then simplified by removing redundancy of enrich terms with the simplify function with cutoff $=0.7$, by $=$ p.adjust and measure $=$ Wang options. Top GO terms for each sample were selected by adjusted $P$ values .

\section{Statistics}

Results are presented as mean \pm SEM. A 2-tailed Student $t$ test was used to compare the differences between 2 groups. One-way ANOVA was used for simple multiple comparisons. Two-way ANOVA was used for studies with 2 independent variables. A post hoc test with Tukey correction was applied to multiple comparisons. Statistical significance was defined as $P<0.05$. Statistical analyses were performed using GraphPad Prism 9 software.

\section{Study approval}

Animal studies were approved by the Institutional Animal Care and Use Committee of Case Western Reserve University.

\section{Author contributions}

XL, EC, XT, IW, and RZ conducted experiments and acquired and analyzed data. HWJ and RHA performed bioinformatics. XL and EC designed the research studies. MKJ supervised the project. XL and MKJ wrote the manuscript with input from all authors.

\section{Acknowledgments}

This work was supported by American Heart Association (AHA) Transformative Project Award 18TPA34230033 (to XL), NIH grant 1R35HL135789, an Elisabeth Severance Prentiss Foundation grant, the AHA - Paul G. Allen Frontiers Group grant 19PABHI34580006 (to MKJ), and the Leducq Foundation grant 18CVD03 (to RHA and MKJ). This research was supported by the Cytometry \& Imaging Microscopy Shared Resource of the Case Comprehensive Cancer Center (grant P30CA043703), Case Western Reserve University School of Medicine. The authors acknowledge the Flow Cytometry Core and the Genomics Core at the Cleveland Clinic Lerner Research Institute for cell sorting and 10× single-cell omics services.

Address correspondence to: Mukesh K. Jain, Case Cardiovascular Research Institute, 10900 Euclid Ave., Cleveland, Ohio 44106, USA. Phone: 216.368.3607; Email: mxj84@case.edu.

1. Kurisu S, Sato H. [History of cardiology in the last 100 years: Japanese contribution to studies on Tako-tsubo like left ventricular dysfunction]. Nihon Naika Gakkai Zasshi. 2002;91(3):849-852.

2. Akashi YJ, et al. Epidemiology and pathophysiology of Takotsubo syndrome. Nat Rev Cardiol. 2015;12(7):387-397.

3. Tornvall P, et al. A case-control study of risk markers and mortality in Takotsubo stress cardiomyopathy. J Am Coll Cardiol. 2016;67(16):1931-1936.

4. Redfors B, et al. Mortality in takotsubo syndrome is similar to mortality in myocardial infarction - A report from the SWEDEHEART registry. Int J Cardiol. 2015;185:282-289.

5. Medina de Chazal H, et al. Stress cardiomyopathy diagnosis and treatment: JACC state-of-the-art review. J Am Coll Cardiol. 2018;72(16):1955-1971.

6. Templin C, et al. Clinical features and outcomes of Takotsubo (stress) cardiomyopathy. N Engl J Med. 2015;373(10):929-938

7. Isogai T, et al. Early beta-blocker use and in-hospital mortality in patients with Takotsubo cardiomyopathy. Heart. 
2016;102(13):1029-1035.

8. Hurst RT, et al. Takotsubo cardiomyopathy: a unique cardiomyopathy with variable ventricular morphology. JACC Cardiovasc Imaging. 2010;3(6):641-649.

9. Wittstein IS, et al. Neurohumoral features of myocardial stunning due to sudden emotional stress. N Engl J Med. 2005;352(6):539-548.

10. Marcovitz PA, et al. Pheochromocytoma presenting with Takotsubo syndrome. J Interv Cardiol. 2010;23(5):437-442.

11. Eliades M, et al. Takotsubo cardiomyopathy associated with thyrotoxicosis: a case report and review of the literature. Thyroid. 2014;24(2):383-389.

12. Elgendy AY, et al. Clinical presentations and outcomes of Takotsubo syndrome in the setting of subarachnoid hemorrhage: a systematic review and meta-analysis. Eur Heart J Acute Cardiovasc Care. 2018;7(3):236-245.

13. Khwaja YH, Tai JM. Takotsubo cardiomyopathy with use of salbutamol nebulisation and aminophylline infusion in a patient with acute asthma exacerbation. BMJ Case Rep. 2016;2016:bcr2016217364.

14. Wilson HM, et al. Characterization of the myocardial inflammatory response in acute stress-induced (Takotsubo) cardiomyopathy. JACC Basic Transl Sci. 2018;3(6):766-778.

15. Scally C, et al. Myocardial and systemic inflammation in acute stress-induced (Takotsubo) cardiomyopathy. Circulation. 2019;139(13):1581-1592.

16. Nef HM, et al. Tako-Tsubo cardiomyopathy: intraindividual structural analysis in the acute phase and after functional recovery. Eur Heart J. 2007;28(20):2456-2464.

17. Gomez Perdiguero E, et al. Tissue-resident macrophages originate from yolk-sac-derived erythro-myeloid progenitors. Nature. 2015;518(7540):547-551.

18. Cohen HB, Mosser DM. Cardiac macrophages: how to mend a broken heart. Immunity. 2014;40(1):3-5.

19. Epelman S, et al. Embryonic and adult-derived resident cardiac macrophages are maintained through distinct mechanisms at steady state and during inflammation. Immunity. 2014;40(1):91-104.

20. Lavine KJ, et al. Distinct macrophage lineages contribute to disparate patterns of cardiac recovery and remodeling in the neonatal and adult heart. Proc Natl Acad Sci U S A. 2014;111(45):16029-16034

21. Liao X, et al. Distinct roles of resident and nonresident macrophages in nonischemic cardiomyopathy. Proc Natl Acad Sci U S A. 2018;115(20):E4661-E4669.

22. Dick SA, et al. Self-renewing resident cardiac macrophages limit adverse remodeling following myocardial infarction. Nat Immunol. 2019;20(1):29-39.

23. Kneale BJ, et al. Gender differences in sensitivity to adrenergic agonists of forearm resistance vasculature. $J$ Am Coll Cardiol. 2000;36(4):1233-1238.

24. Ohradanova-Repic A, et al. Extracellular purine metabolism is the switchboard of immunosuppressive macrophages and a novel target to treat diseases with macrophage imbalances. Front Immunol. 2018;9:852.

25. Soberman RJ, Karnovsky ML. Metabolism of purines in macrophages. Effect of functional state of the cells. J Exp Med. 1980;152(1):241-246.

26. Hulsmans M, et al. Macrophages facilitate electrical conduction in the heart. Cell. 2017;169(3):510-522.

27. Cramer T, et al. HIF-1alpha is essential for myeloid cell-mediated inflammation. Cell. 2003;112(5):645-657.

28. Nayak L, et al. The thromboprotective effect of bortezomib is dependent on the transcription factor Kruppel-like factor 2 (KLF2). Blood. 2014;123(24):3828-3831.

29. Li W, et al. Cardiovascular and thrombotic complications of novel multiple myeloma therapies: a review. JAMA Oncol. 2017;3(7):980-988.

30. Yannaki E, et al. The proteasome inhibitor bortezomib drastically affects inflammation and bone disease in adjuvant-induced arthritis in rats. Arthritis Rheum. 2010;62(11):3277-3288.

31. Lassoued S, et al. Bortezomib improved the joint manifestations of rheumatoid arthritis in three patients. Joint Bone Spine. 2019;86(3):381-382.

32. Liu J, et al. Bortezomib followed by autologous stem cell transplantation in a patient with rheumatoid arthritis: a case report and review of the literature. Medicine (Baltimore). 2016;95(52):e5760

33. van der Heijden JW, et al. The proteasome inhibitor bortezomib inhibits the release of NFkappaB-inducible cytokines and induces apoptosis of activated T cells from rheumatoid arthritis patients. Clin Exp Rheumatol. 2009;27(1):92-98.

34. Walsh RC, et al. Proteasome inhibitor-based primary therapy for antibody-mediated renal allograft rejection. Transplantation 2010;89(3):277-284

35. Mohty M, et al. Effects of bortezomib on the immune system: a focus on immune regulation. Biol Blood Marrow Transplant. 2013;19(10):1416-1420.

36. Bajpai G, et al. Tissue resident CCR2- and CCR2+ cardiac macrophages differentially orchestrate monocyte recruitment and fate specification following myocardial injury. Circ Res. 2019;124(2):263-278

37. Scanzano A, Cosentino M. Adrenergic regulation of innate immunity: a review. Front Pharmacol. $2015 ; 6: 171$.

38. Forte E, et al. Type $2 \mathrm{MI}$ induced by a single high dose of isoproterenol in C57BL/6J mice triggers a persistent adaptive immune response against the heart. J Cell Mol Med. 2021;25(1):229-243.

39. Mahabeleshwar GH, et al. The myeloid transcription factor KLF2 regulates the host response to polymicrobial infection and endotoxic shock. Immunity. 2011;34(5):715-728.

40. Liao X, et al. Krüppel-like factor 4 regulates macrophage polarization. J Clin Invest. 2011;121(7):2736-2749.

41. Liao X, et al. Kruppel-like factor 4 is critical for transcriptional control of cardiac mitochondrial homeostasis. J Clin Invest. 2015;125(9):3461-3476

42. Shao Y, et al. Novel rat model reveals important roles of $\beta$-adrenoreceptors in stress-induced cardiomyopathy. Int J Cardiol. 2013;168(3):1943-1950

43. Shao Y, et al. A mouse model reveals an important role for catecholamine-induced lipotoxicity in the pathogenesis of stress-induced cardiomyopathy. Eur J Heart Fail. 2013;15(1):9-22.

44. Liao X, et al. Krüppel-like factor 4 regulates pressure-induced cardiac hypertrophy. J Mol Cell Cardiol. 2010;49(2):334-338. 